\title{
TeV to PeV neutrinos and gamma-rays with Mountain SHALON Mirror Cherenkov Telescope.
}

\author{
V. G. Sinitsyna, T. P. Arsov, S. S. Borisov, F. I. Musin, \\ S. I. Nikolsky, V. Y. Sinitsyna and G. F. Platonov \\ P. N. Lebedev Physical Institute, Leninsky pr. 53, Moscow, Russia \\ Presenter: V. G. Sinitsyna (sinits@ sci.lebedev.ru), rus-sinitsina-VG-abs4-og25-poster
}

The detection of Extraterrestrial Very High Energy Neutrinos by Atmospheric Cherenkov Telescopic System SHALON is discussed. The analysis of results of observation of extensive air showers at height of $3338 \mathrm{~m}$ above the sea level by gamma-telescope SHALON at the zenith angles $72^{\circ}, 76^{\circ}, 84^{\circ}, 97^{\circ}$ are presented. The observation results are compared with the data of detection of showers according to the direction into the zenith. The observation has been carried out at Tien -Shan station with SHALON gamma-telescope. The SHALON telescopic system has mirror with area of $11.2 \mathrm{~m}^{2}$ and 144 PMT matrix with angular resolution of experimental method of $0.1^{\circ}-0.2^{\circ}$, and the most in the world angular size of $>8^{\circ}$. It allows to control the background of cosmic ray particle emission and the atmospheric transparency continuously with observation that means the increasing of observation efficiency. So it is the telescope characteristics that permit to start the search of local neutrino sources with energy $10^{13}-10^{16} \mathrm{eV}$ on EAS generating in mountain-range located at some 7 and more kilometers from gamma-telescope (in Russian the SHALON abbreviation means - the Extensive Air Showers from Neutrino).

\section{Introduction}

The gamma-source search is the first step of the astronomy to the very high energy range. Only neutrino astronomy in the future can complete the search and investigation of galactic and metagalactic objects where the proton and nucleus acceleration processes are realized. These processes accompanied by generation of gammaquanta and neutrino which are not scattered in Universe magnetic field, that gives an addition possibilities of investigation of the properties of active stellar objects at a very high energy. The observations have been carried out on P. N. Lebedev Physical Institute Tien - Shan high-mountainous station with gamma-telescope SHALON-1 since 1992 and started operating SHALON-2 [1 - 5].

\section{The Observation of Cherenkov Bursts at the Zenith Angle $97^{\circ}$}

UHE neutrinos may be searched in observations at large zenith angle. Because of mountain valley screens from background showers, the only particles that can survive are neutrinos with energy $>10^{13} \mathrm{eV}$, come under the horizon, interact with mountain matter and product of the interaction produce an extensive air showers. In actual conditions the mirror telescope placement (Fig. 1) the distance till the opposite slope of the gorge is $\sim 7 \mathrm{~km}$ or $\sim 16.5$ radiation units of length, that is quite enough for the development of an electromagnetic cascade till the structure characteristic for the rarefied atmosphere. The purpose of observations was revealing of background conditions when anthropogenic sources of light are absent. During 206 hours of observations 5 events were detected (Fig. 2a) which have expected angular characteristics of a light burst of an electron-photon cascade developing within a telescope observation angle (Fig. 2b). These showers have energy in the range of about $5-15 \mathrm{TeV}$. All other 265 events of detection of short-range light bursts in the atmosphere have not a narrow angle light direction and are chaotically distributed along the whole matrix or its part of a light-receiver (Fig. 1). These events may be interpreted as a reflection of a Cherenkov burst from a snow mountain slope or 


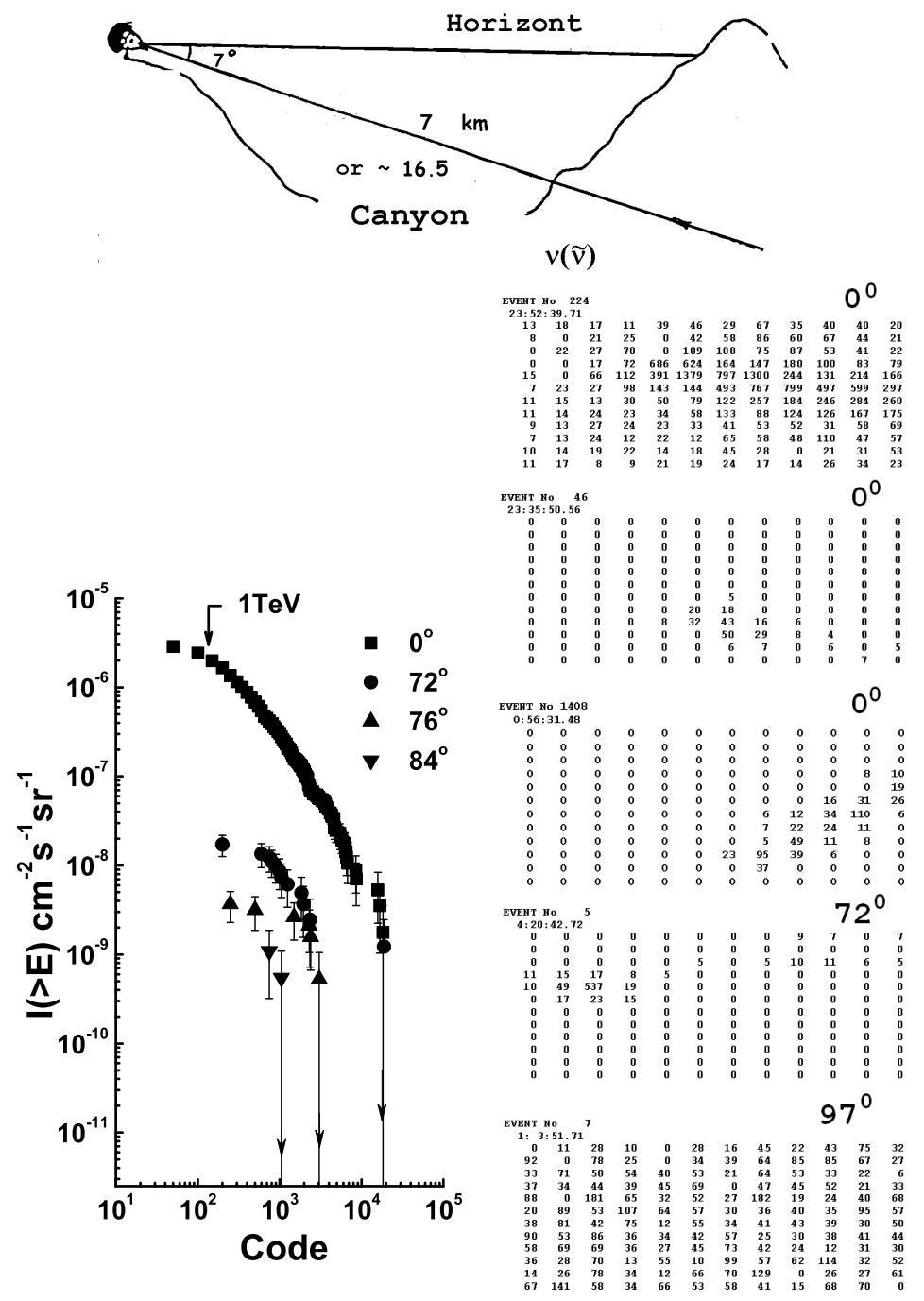

Figure 1. The geometry of subhorizontal observation sessions. The spectrum of extensive air showers Cherenkov radiation by telescope SHALON observation within $8^{\circ}$ full angle. PMT amplitude arbitrary units are laid off along the abscissa.

as an ionization luminescence of the atmosphere while an extensive air showers transition within a telescope observation angle.

\section{Extensive Air Showers under a Large Zenith Angle}

The observation of extensive air showers under a large zenith angle attracts the investigator attention at least by two reasons. On the one hand, the cascade development till the maximum of its development in a rarefied atmosphere changes the structure and properties of extensive air showers. On the other hand, the possibility 


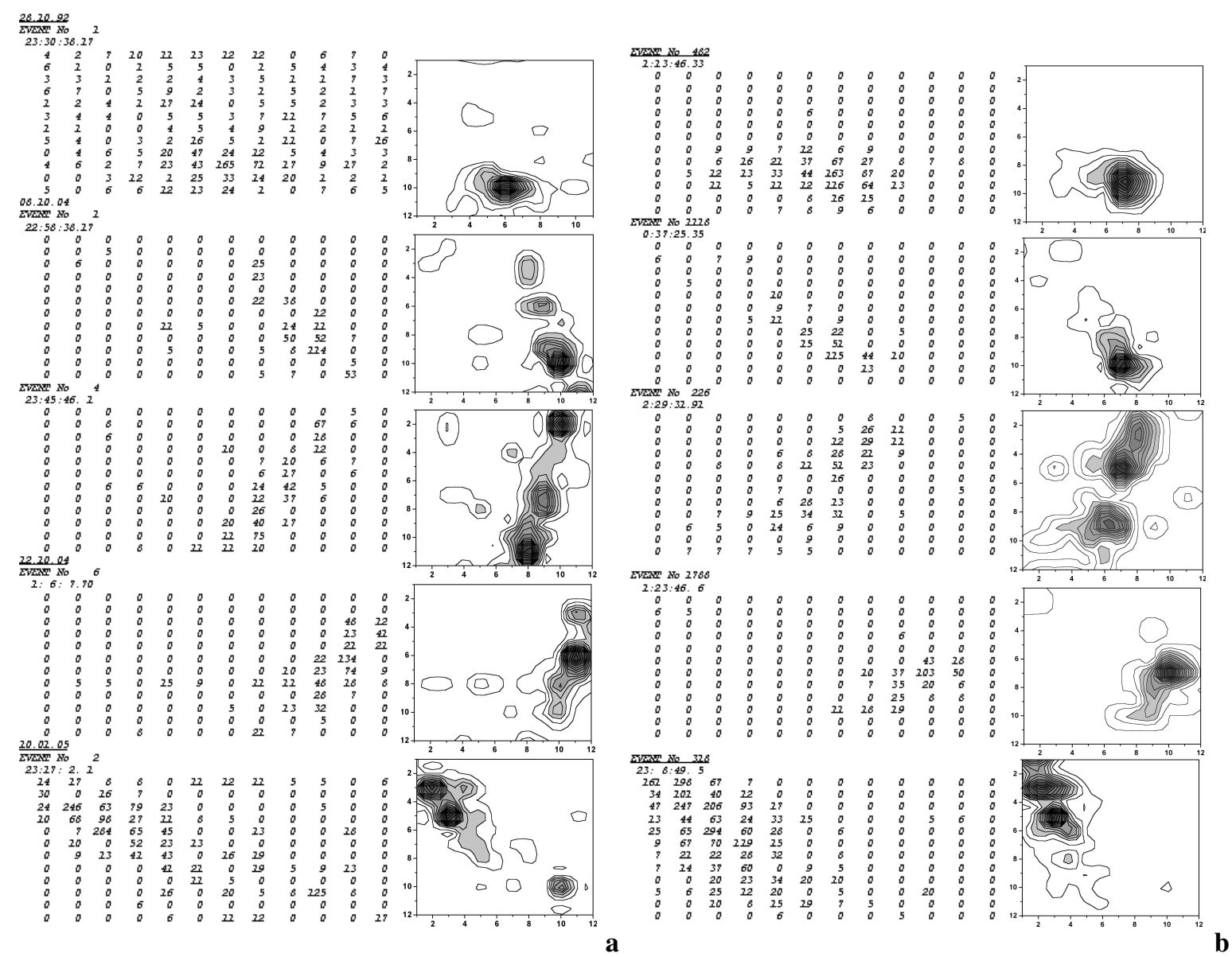

Figure 2. a - Cherenkov Radiation of Extensive Air Showers Observed at $97^{\circ}$ Zenith Angles by SHALON; b - Cherenkov Radiation of Extensive Air Showers Observed at $0^{\circ}$ Zenith Angles by SHALON

to observe showers by means of simple of systems of wide-angle detectors of Cherenkov radiation separated at large distance seems attractive. A mirror telescope SHALON due to the trigger control of the detection of bursts of a short-range $(8 \mathrm{nsec})$ signal in $\sim 4$ photomultipliers of the light-receiver matrix allows one to know the number of observed bursts without observation of conditions of an angular picture of a Cherenkov burst. While observing into the zenith the number of such bursts is similar to the number of evens, identified as a Cherenkov burst from an extensive air shower radiating in the atmosphere at the distance less than $10 \mathrm{~km}$ above the telescope. In observation by the telescope directed at the mountain slope under a zenith angle $\sim 97^{\circ}$ 5 events were detected (Fig. 2). While observing under a smaller zenith angles a fraction of such events presented at Fig. 1 increases. However, one should point out, that a number of detected showers within a telescope observation angle decreases while increasing of a zenith angle more considerably than it is expected while neglecting by absorption and dissipation of Cherenkov photons in the atmosphere (10 times at the angle $\left.76^{\circ}\right)$.

Carrying out of the observation allows one to make the following conclusion. At first, a night star sky doesn't produce any background events, preventing the observations of electron-photon cascades coming from under 
the earth surface. Secondary, the observation of Cherenkov bursts from extensive air showers under the large zenith angles, for example using of horizontal extensive air showers for investigation of an energy spectrum of ultra-high energy cosmic rays is complicated by absorption of Cherenkov photons by a large atmosphere thickness.

\section{Conclusion}

It is supposed to overcome the main difficulty of observation of EAS, generated by neutrino in conditions of high mountainous observations, connected with the small cross section of neutrino-nuclei inelastic collisions. Two facts allow to carry out the search experiments. The hadron cascades with energy $>10^{13} \mathrm{eV}$ are generated by neutrino in the ground of mountain on the thickness $<300 \mathrm{~g} / \mathrm{cm}^{2}$. The Cherenkov radiation of the hadron cascades will be observed along the direction of neutrino by gamma-telescope placed on the distance about $7 \mathrm{kms}$ from a mountain slope in area of more than $7 \times 10^{5} \mathrm{~m}^{2}$. These cascades look like the usual extensive air showers generated in atmosphere with narrow light shape. Presently, the fluxes of galactic gamma-quantum sources Cygnus X-3, Tycho's SNR, Geminga of $<10^{-14} \mathrm{~cm}^{-2} \mathrm{~s}^{-1}$ are observed by SHALON. The appearing of one shower per $>100$ observation hours is expected if the flux of neutrino from local sources is

$10^{-15} \mathrm{~cm}^{-2} \mathrm{~s}^{-1}[1-12]$. During 206 hours of observations 5 events were detected which have expected angular characteristics of a light burst of an electron-photon cascade developing within a telescope observation angle.

\section{References}

[1] Nikolsky S. I., Sinitsyna V. G., VANT Ser. TFE. 1331 (1987) 30.

[2] Sinitsyna V. G., Toward a Major Atmospheric Cherenkov Detector -II, ed. Lamb R. C. (Iowa State University) (1993) 91.

[3] Sinitsyna V. G., Nuovo Cimento 19C(6) (1996) 965.

[4] Sinitsyna V. G., Nikolsky S. I., et.al., Nucl. Phys. B (Proc. Suppl.) 75A (1999) 352; 97, (2001) 215, 219.

[5] Nikolsky S. I. and Sinitsyna V. G., Physics of Atomic Nuclei 67(10) (2004) 1900.

[6] Klapdor-Kliengrothaus H.V., Zuber K., in Teilchenastrophysik, ed. Teubner B.G. (GmbH, Stuttgart, 2000).

[7] Resvanis L.K., Nucl. Phys. B (Proc. Suppl.) 122, 24 (2003).

[8] Degrange B., Toward a Major Atmospheric Cherenkov Detector-I, ed. Fleury P., Vacanti G. (Frontieres) (1992) 171.

[9] Bugaev E.V. and Shlepin Yu. V., Nucl. Phys. B (Proc. Suppl.) 122, 341 (2003).

[10] Fargion D., et. al., ApJ 613, 1285 (2004).

[11] Berezinsky V.S., Gazaev A.Z., JETP lett 25(5) (1977) 276.

[12] Zatsepin G.T. and Berezinsky V.S., Neutrono Astrophysic Problems 2 (INR, Russian Academy of Science, 1980) 172. 